\title{
Scanning Electron Microscopy (SEM) Study Showed the Mode Action of Metarhizium anisopliae on Odontotermes obesus
}

\author{
Pranab Dutta*, J. Pegu, H. Kaushik, P. Kaman and Ankita Das
}

Department of Plant Pathology, Assam Agricultural University, Jorhat-785013, Assam, India

*Corresponding author

\section{A B S T R A C T}

\section{Keywords}

Metarhizium anisopliae, Odontotermes obesus,

Scanning Electron

Microscopy

Article Info

Accepted:

10 September 2018

Available Online:

10 October 2018

The present study was done to investigate the histopathological effect of the entomopathogenic fungi, Metarhizium anisopliae on Odontotermes obesus using Scanning Electron Microscopy (SEM). Adult worker termites were inoculated with $1 \times 10^{9}$ conidia $/ \mathrm{ml}$ suspension of the fungi and maintained at $25 \pm 1{ }^{\circ} \mathrm{C}$ temperature and $80 \%$ relative humidity. SEM study showed that when this mitotic (asexual) spore (called conidia) of the fungus came into contact with the body of the termite, they germinated and the hyphae of the fungus that emerged penetrated the cuticle. The hyphal penetration of the fungi through the insect cuticle as well as proliferation formed a dense network of mycelium in the insect cuticle. This fungus also invaded the host through the integument and caused death of the insect. This lethal effect to the insect is very likely to be aided by the production of insecticidal cyclic peptides (destruxins) by the fungus. SEM study revealed that it is a convenient tool to observe the mode of action of entomopathogenic fungi and to observe how they are able to colonize and infect the host.

\section{Introduction}

Termites are one of the most important pest of agricultural, horticultural, plantation crops, forest trees etc. Conventional termite control depends largely on the use of chemical insecticides. However many of these insecticides are banned now a days because of its ill effects to non-target insects as well as their persistence in the environment.

Due to increasing cost of the pesticides, outbreak of secondary pests, insecticidal resistance and pollution problems search for alternatives to chemical pest control has assumed greater importance in recent years.
One such alternative method is biological control using entomopathogenic fungi (Khan et al., 1993). Termites are considered as good candidate for control with the entomopathogenic organisms because they live in a conducive environment, humid, minimal diurnal temperature, fluctuations, crowded and with considerable social interaction (Delante et al., 1995; Creffield, 1996). Biological control of termites using entomopathogenic fungi have been reported by Lai et al., 1982; Hanel and Watson, 1983; Gurusubramanian, et al., 1999; Rath, 2000.

The scanning electron microscope (SEM) uses a focused beam of high-energy electrons to 
generate a variety of signals at the surface of solid specimens. The signals that derive from electron-sample interactions reveal information about the sample including external morphology (texture), chemical composition and crystalline structure and orientation of materials making up the sample. The objective of the present investigation was to study the external development of Metarhizium anisopliae treated on the termite body using SEM.

\section{Materials and Methods}

\section{Rearing of termites}

Adult workers of Odontotermes obesus (Termites) were collected from the Experimental Garden for Plantation Crops (EGPC), Assam Agricultural University, Jorhat. Collected termites were reared at room temperature in plastic containers with sand and wetted corrugated cardboard until use.

\section{Source of fungal isolate}

The pure culture of the fungus, Metarhizium anisopliae was obtained from the insect isolates by Pegu et al., (2012). These fungi were then sub cultured in Potato Dextrose Agar (PDA) media and incubated at $25 \pm 1^{\circ} \mathrm{C}$ for 5-7 days. Twenty (20) $\mathrm{ml}$ of the sterilized PDA media was poured aseptically in sterilized petridishes. On solidification of the media the petridishes were inoculated with a loop full of fungal mass from the pure culture and then incubated at $25 \pm 1^{\circ} \mathrm{C}$ for sporulation. Isolated and purified cultures were maintained by periodical transferring in fresh PDA slants.

\section{Fungal suspensions}

Fungal propagules were scrapped out from the surface of the mass culture with the help of a fine brush. A stock suspension was prepared by placing harvested propagules in pure distilled water. Homogenous suspension of viable conidia was filtered through double layered muslin cloth to remove the mycelial mat and suspended in 0.05 per cent aqueous Tween 80. A Neubeur Haemocytometer was used to estimate the conidial concentration and subsequent appropriate dilutions were made thereof.

\section{Bioassay}

Twenty numbers of termites were transferred to petridishes lined with filter paper. Termites were inoculated with a suspension $1 \times 10^{6}$ conidia/ml of $M$. anisopliae and maintained at $25 \pm 1{ }^{\circ} \mathrm{C}$ and $80 \%$ relative humidity. The termite mortality was observed at $24 \mathrm{~h}$ intervals for up to 7 days. Dead termites were incubated in a humid chamber to confirm growth of the fungus on their cadavers. A batch of uninfected termites was maintained as a control.

\section{Preparation of treated termite samples for scanning electron microscopic examination}

The infected death termites were held in absolute ethanol for one month. It was checked periodically so that the alcohol does not evaporate from the container.

The insect was then removed, washed gently with absolute ethanol and allowed to dry in a covered dish (to avoid dust) for at least one week.

This step allows the alcohol that has replaced the insects' blood to evaporate. Then, the insect was placed on a mount on a spot of carbon paint to hold it in place and coated with a layer of gold. Samples were observed under SEM (JEOL, JSM- 6390LV) and photographs were taken to get detailed morphological changes of the insect body at Department of Physics, Tezpur University, Assam. 


\section{Results and Discussion}

At low magnification the infected termite showed distortion and deformation of the whole body having no proper orientation. Distortion was more in abdominal part of the insect body. There was shrinkage in the head cuticle and deformity of head sensilla (Fig. 1bc). At higher magnification the fungal colonies were clearly visible throughout the body parts (Fig. 1d). The spores were characterized by green colour. The fungal hyphae together formed a dense network of mycelium. The spores and hyphae of the fungus were also seen in the body cavity.

The formation of haloes around fungal conidia and germination tubes on the insect cuticle was also observed with conidia adhesion and germination. A thickening of the extremity of the germ-tube, characterizing the formation of aspersoria of the fungus was also observed during penetration by the fungus. The mycelial extrusion of $M$. anisopliae was more in the intersegmental areas resulting in a process of cuticle degradation along the whole body of the insect. The whole body was covered by $M$. anisopliae conidia.

Scanning electron microscopy (SEM) of termite treated with the fungus, $M$. anisopliae clearly revealed adhesion and penetration structures in the infected termite. Infection of termite by the fungus, $M$. anisopliae requires adhesion, penetration into the host (St Leger, 1993) and establishment of the pathogen in the host (Charnley, 1989; Sampson et al., 1988). Penetration through the host cuticle is the mode of entry for most entomopathogenic fungi (Charnley, 1984). During fungal infection, the first step prior to penetration is the adhesion of fungi to the host cuticle (Boucias and Pendland, 1991). Fragues (1984) suggested adhesion to occur at three successive stages: adsorption of the fungi propagules to the cuticular surface, adhesion of the interface between propagules and epicuticle and fungi germination and development at the insect cuticular surface, until appresoria are developed to start the penetration stage. $M$. anisopliae produces some cyclic peptide toxins, destruxins, which exhibit a variety of insecticidal actions by Sharif et al., (2010) and thirty-eight destruxin analogues have been reported to date (Schrank and Vainstein, 2010).

The formation of haloes on the insect cuticle at the germination and penetration points was also observed. The occurrence of these haloes may be due to production and excretion of exoenzymes by the entomopathogen during the infective process. The combination of proteolitic enzymes and chitinase produced by the fungal mycelium may digest the insect cuticle, facilitating the penetration of the insect integument. Once the fungus penetrates the host; it produces toxins that overcome the insect immune system.

Appressoria of $M$. anisopliae was also formed in the body of the infected termite. This was characterized by a thickening of the extremity of the germ-tubes. This Thickening is probably due to the translocation of the conidial cytoplasmatic content to facilitate the enzymatic synthesis necessary for the penetration phase. In some areas such as the insect head, extensive growth of germ-tubes was observed, probably due to resistance to fungal penetration in these areas with more heavily sclerotized cuticle. The formation of haloes on the insect cuticle at the germination and penetration points was also observed. An enzyme of the chymoelastase group (Pr1) has been implicated in cuticle degradation (St. Leger et al., 1988). According to these authors, M. anisopliae uses up to $78 \%$ of the protein synthesis for the production of this important enzyme in the process of penetration. Pr1 is a key factor in the virulence of this fungus. 
Fig.1 SEM image of Metarhizium anipliae infected termite (a-d)
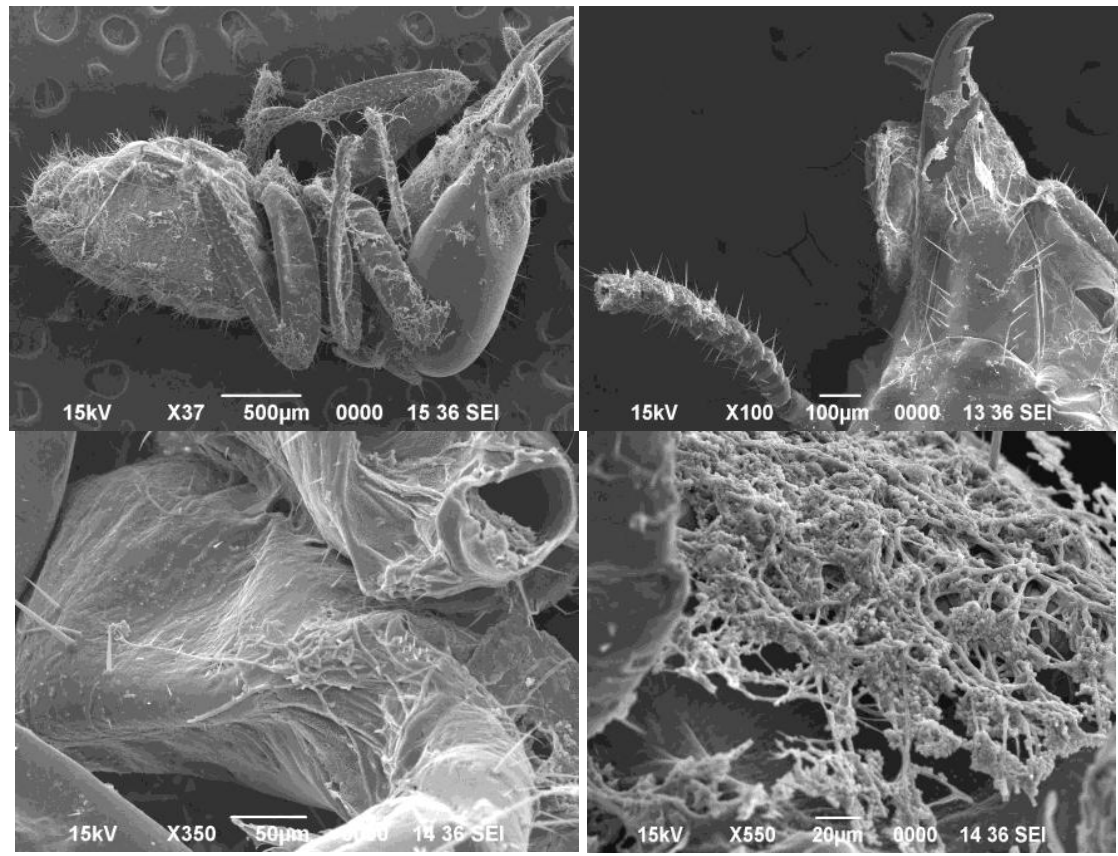

a. M. anisopliae infected adult termite, b-c. Shrinkage and deformity in the cuticle and body parts, d. Growth of fungal structure arising from infected termite body

Despite the standardization in fungal application, the inoculums that reach the insects are variable. This variation may interfere with the extent of colonization of the insect, the speed of mycelium growth, and conidiogenesis, resulting in differences in the mortality of the insects.

The rates of conidia germination and penetration of the insect integument have been shown to be related to the virulence of the fungi and the susceptibility of the insect host (Pekrul \& Grula, 1979; Fargues, 1984). Fungal infection by $M$. anisopliae on the termite body begins when conidia (asexual spores) attach to insect's cuticle.

The spores germinate and penetrate the insect's skin and enter the host. Once the fungus penetrates the host, it produces toxins that overcome the insect immune system. Thereafter, the hyphae penetrate through the cuticle to the outside and cause green sporulation on the insect's body.
M. anisopliae has a high potential for exploitation into a commercial bio insecticide in India. Their low toxicity to humans compared with chemical pesticides is an advantage, especially in India, where the risk of misuse of pesticides is very high. Further studies should include optimisation of dosage for field application and treatment of large area in different agro ecological zones, and socioeconomics. In conclusion, the results obtained in these experiments establish the pathogenicity of entomopathogenic fungi $M$. anisopliae on termite as biological control agent. Biological control with pathogenic fungi is promising alternative to chemical control against the termites.

\section{Acknowledgment}

The authors acknowledged the help, guidance and continuous support received from Dr. C. Hazarika, DPGS, Dr. G. N. Hazarika, Director of Research (Agri), Dr. D.K. Bora, Dean, Faculty of Agriculture, Assam 
Agricultural University, Joraht-13, Assam. The help received from Tezpur University with Sophisticated Equipment Facilities Services is also duly acknowledged.

\section{References}

Boucias DG, Pendland JC. In: Latge P, Boucias DG, editors. In fungal cell wall and immune response. Berlin: Springer; 1991. p. 303-16.

Charnley AK. Mechanisms of fungal pathogenesis in insects. In: Whipps JM, Lumsden RD, editors. The biotechnology of fungi for improving plant growth. London: Cambridge University; 1989. p. 85-125

Charnley, AK. (1984).In invertebratemicrobial interactions. In: Anderson JM, Rayner ADM, Walton DWH, editors. Physiological aspects of destructive pathogenesis by fungi: A speculative review. Brit. Mycol. Soc. Symp. 6. London: Cambridge University Press; p. 229-70.

Creffield J. W. (1996). Wood-destroying insects, Wood Borers and Termites. CSIRO Publishing, Collingwood. 44 pp.

Delante, K. M., Grace J. K. and Tome C. H. M. (1995). Potential use of pathogenic fungi in baits to control the Formosan subterranean termite (Isopt., Rhinitermitidae). J. Appl. Entomol 119, 429-433.

Farges J. In: Roberts DW, Aist JR (Eds.). In infection processes of fungi. Conference Report, The Rockefeller Foundation, NY, 1984. p. 90-110.

Fargues, J. (1984). Adhesion of the fungal spore to the insect cuticle in relation to pathogenicity. In: Roberts, D.W.; Aist, J.R. (Ed.) Infection processes of fungi; conference report. New York: The Rockfeller Foundation. pp. 90-110.

Gurusubramanian, G., K.G. Rajeeb and A.K. Tamuli. (1999). Susceptibility of
Odontotermes obesus Rambur to entomogenous white muscardine fungus Beauveria bassiana (Bals.) Vuill (Deuteromycotina: Hyphomycetes). Insect Sci. Appl., 19, 57-65.

Hanel, H., Watson, J.A.L. (1983). Preliminary field tests on the use of Metarhizium anisopliae for control of Nasutitermes exitiosus (Hill) (Isoptera: Termitidae). Bulletin of Entomological Research 73, 305-313.

Khan, H. K.; Jayaraj, S. and Gopalan, M. (1993). Muscardine fungi for the biological control of agro-forestry termite, Odontotermes obesus (Rambur). J. Bio. Contl. 4: 122-123.

Lai, P.Y., Tamashiro, M. and Fuji, J.K. (1982). Pathogenecity of six strains of entomogenous fungi for Coptotermes formosanus. J. Invertebr. Pathol. 39, 15.

M. Mohammadi Sharif, A.R. Hadizadeh and M.A. Tajick Ghanbary. (2010). Evaluating Toxicity of Extracted Destruxin from Metarhizium anisopliae Against Citrus Leafminer, Phyllocnistis citrella. American J. Envt. Sci. 6 (4): 379-382.

Pekrul, S.; Grula, E. A. (1979). Mode of infection of the corn earworm (Heliothis zea) by Beauveria bassiana as revealed by scanning electron microscopy. J. Invertebr Pathol. 34, p.238-247

Rath A. C. (2000). The use of entomopathogenic fungi for control of termites. Biocont Sci.Tech. 10, 563581.

Sampson RA, Evans HC, Latge JP. (1988). Atlas of entomopathogenic fungi. Berlin: Springer

Schrank, A, Vainstein, MH. (2010). Metarhizium anisopliae enzymes and toxins. Toxicon 56:1267-1274.

St. Leger RJ, Durrands, PK, Charnley, AK and Cooper, RM. (1988). Role of extracellular chymoelastase in the 
virulence of Metarhizium anisopliae for Manduca sexta. J. Invertebr. Pathol. 52:285-293.

St. Leger RJ. Biology and mechanisms of invasion of deuteromycete fungal pathogens. In: Beckage NC, Thompson SN, Federici BA, editors. Parasites and ARTICLE IN PRESS 34 C. Dong et al., pathogens of insects, vol. 2. New York: Academic Press; 1993. p. 211-29.

\section{How to cite this article:}

Pranab Dutta, J. Pegu, H. Kaushik, P. Kaman and Ankita Das. 2018. Scanning Electron Microscopy (SEM) Study Showed the Mode Action of Metarhizium anisopliae on Odontotermes obesus. Int.J.Curr.Microbiol.App.Sci. 7(10): 901-906.

doi: https://doi.org/10.20546/ijcmas.2018.710.100 